\title{
Information video analytics system for the prevention of physical inactivity in students
}

\author{
I.O. Egorochkina ${ }^{1 *}$, E.S.Tsygankova ${ }^{1}$, E.A. Shlyakhova ${ }^{1}$, I.A. Serebryanaya ${ }^{1}$, and D.S. \\ Serebryanaya ${ }^{2}$ \\ ${ }^{1}$ Don State Technical University, 344002, Rostov-on-Don, Russia \\ ${ }^{2}$ South Russian Institute of Management branch of the Russian Academy National Economy and \\ Public Administration under the President of the Russian Federation, 344000, Rostov-on-Don, Russia
}

\begin{abstract}
The project "SportTrainer" is presented - an information system of video analytics and personal recommendations for the prevention of hypo-dynamics and increasing the effectiveness of personal training of students who want to achieve a certain sports result and improve their figure. The developed system "SportTrainer" realizes consulting on training programs, nutrition, provides control over the implementation of the selected programs and the achievement of goals. A digital trainer can suggest how effective the selected program is for a particular individual, selects the necessary exercises, make adjustments to the training program and diet, simulate and visualize progress. In the research work, modern technologies of video analytics, computer vision, machine learning and video streaming were used, which allow real-time processing of a video stream, analysis and pattern recognition, and fully automate the process of personal training at home. The implementation of the developed activities of the "SportTrainer" project was carried out in three control groups, represented by bachelors, masters and teachers. Statistical processing of basic indicators and results achieved in the process of testing the "SportTrainer" system has been carried out. The presented statistical data confirm the effectiveness of the program within the control training groups. The developed system is applicable to a wide range of the population, effective in conditions of social distancing due to a pandemic situation, since it improves conditions for home, including professional sports. In the future, a sharp increase in their share is expected in comparison with traditional training techniques.
\end{abstract}

\section{Introduction. Relevance of the problem}

Modern students spend most of their study and leisure time sitting at a computer and other gadgets. There is a tendency for a high level of stress tension with a hypodynamic lifestyle [1].

Physical inactivity is not a disease, but it leads to serious disorders in the body [2]. Doctors warn that a sedentary lifestyle is one of the ten leading causes of disability

\footnotetext{
* Corresponding author: arin77@bk.ru
} 
worldwide, and in $90 \%$ of cases it is a trigger for the development of obesity and joint diseases [3].

Obviously, the ways to overcome physical inactivity should be aimed at increasing physical activity in each person.

One of the priority tasks of the sports industry in Russia is to increase by 2024 to $55 \%$ the share of citizens who are systematically involved in physical culture and sports [4].

In accordance with the curriculum, students are required to engage in one of the types of physical activity of their choice once a week. The survey, conducted in the control groups [5] revealed the desire to train more often, including for a certain sports result. The survey also showed [6] that young people practically do not have the opportunity to do additional sports. The lack of proper motivation, convenient time and territory for classes is highlighted as an obstacle [7].

Recently, the number of sports centers and fitness clubs has increased, regular visits to which are an excellent prevention of physical inactivity and body shaping [8]. However, in fitness clubs, trainers often actively engage with clients only if they purchase subscriptions for personal training, otherwise, trainers give only general recommendations. Therefore, $40 \%$ of the respondents would prefer to play sports at home. About $5 \%$ of the respondents regularly do a jog or a simple complex on their own, again referring to the lack of proper motivation.

The creation of intelligent digital avatar programs that provide guidance and personalized training for home workouts will help make a difference. A digital trainer can suggest how effective the selected program is for a particular individual, selects the necessary exercises, make adjustments to the training program and diet, simulate and visualize progress.

As analogs, we analyzed the systems that provide interactive intelligent communication, allowing you to monitor the quality of the exercises performed.

The most commonly used programs include:

- Coach's Eye mobile application focused on tennis, badminton, golf [9];

- Voltathletics is a personal trainer application based on artificial intelligence [10];

- Applications for the implementation of personalized workouts Fitness AI [11];

- Virtuagym [12], etc.

The survey of studies demonstrates the average annual growth of the world market for personal sports systems at $28 \%$ [13]. The development of the personal sports market takes place in the direction of a gradual increase in the analytical capabilities of products, and the use of statistical data, algorithms for modeling individual trajectories of development.

The development of a system of personal recommendations for students' sports is a highly relevant task, especially in a pandemic.

The aim of the research work is to develop an intelligent video analytics system "SportTrainer". The "SportTrainer" is supposed to make the process of doing sports more convenient, efficient and cheaper.

With the "SportTrainer" system, each teacher-mentor, curator of the study group can consult students on training programs, nutrition, provide control over the implementation of the selected programs and the achievement of the set goals.

The developed info-system "SportTrainer" is convenient in conditions of social distancing, since it ensures regularity and increases the effectiveness of home sports, which is important for full-time, part-time students, teachers working remotely.

Unlike existing analogues, we have developed a comprehensive solution that automates the work of a curator-trainer and provides a personalized approach, selection of training programs, nutrition and monitoring the effectiveness of their implementation with the ability to emulate emotional communication, which is aimed at significantly increasing 
motivation for sports. Also, the developed system allows you to work with the accumulated statistics.

\section{Research methods}

In the research work, modern technologies of video analytics, computer vision, machine learning and video streaming were used, which allow real-time processing of a video stream, analysis and pattern recognition, and fully automate the process of personal training at home.

To ensure the development of video analytics algorithms, machine learning technologies based on convolutional neural networks, TensorFlow deep learning framework and high-level ApiKeras were used.

A student can play sports at home with his phone and headphones, while the SportTrainer system analyzes the dynamics of his movements and gives corrective recommendations in a synthesized voice.

To create a recommendatory dialogue system, algorithms for determining the semantic proximity of texts and machine learning technologies were used.

The services Dialog Flow, the Alice platform were used, which will provide a new qualitative level of functioning of dialogue systems, which will ensure the emotional involvement of students, automate many communication functions, and build a system of competitive motivation.

The material for research is formed by "User stories", which include a questionnaire, a bank of measurements of anthropometric data, medical analyzes and a therapist's opinion on the state of health, a food diary.

The toolkit are programs for the interpretation of medical analyzes, programs for silhouette modeling, programs for analyzing the regime of the day, food diary, and physical activity. The methodology for collecting and processing data is presented in $[14,15]$. The technical implementation of the system being developed will be carried out through a typical application interface that recognizes human movements and compares them with a standard.

To implement the project of the "SportTrainer" system, modern software development tools and machine learning technologies were used [16, 17].

To implement the project, the "SportTrainer" system requires the following design requirements:

- The movement tracking module should implement mathematical models of training methods.

- An editor module must be implemented to train models of reference movements.

- The system being developed must ensure the delimitation of access to data depending on the role of the user.

- The system architecture should be developed based on the microservices model.

- The system must support fault tolerance and scalability.

- The mechanism for converting speech to text should be based on Speech Kit and Yandex Alice technologies.

- Speech synthesis should be carried out in real time.

- The functioning of the dialogue system should be based on the Dialog Flow service, to highlight semantically significant elements of phrases and a description of the dialogue script.

- For the development of server architecture and API programming interface, NGNIX and REST API technologies must be used. 
- Development of the server side of the system should be based on Python, Django and deep learning tools for neural network models Tensorflow, Keras.

- Postgre SQL DBMS and Django ORM should be used to implement databases.

- Data transmission should be carried out using the HTTP2 protocol and Web Sockets technology.

\section{Experimental results}

The implementation of the developed activities of the "Sport Trainer" system was carried out in three control groups, represented by bachelors, masters and teachers.

The study involved 125 people aged 19 to 54 years, including: 50 students of engineering, 50 masters and 25 people -university teachers aged 30 to 54 years.

The initial data were analyzed:

Personally questionnaire;

Bank of measurements of anthropometric data;

Silhouette modeling programs;

Interpretation programs for medical tests;

Programs for analyzing the regime of the day, food diary, motor activity.

To study stress resistance indicators, the Boston stress resistance test was used: "Life style analysis questionnaire", the method of which is presented in [14], as well as data from a questionnaire survey to assess the level of physical activity of DSTU students as part of a standard screening examination for base of AVITA DSTU.

Results of the questionnaire:

- in a sitting position spend: $7-8$ hours $-20.3 \%$ of men and $29.8 \%$ of women; $5-6$ hours $9.1 \%$ of men and $40.2 \%$ of women; $4-5$ hours $-16.7 \%$ of men and $13.6 \%$ of women;

- about $60 \%$ of young people do not go in for physical education / sports; about $20 \%$ do spontaneously, about $25 \%$ do it regularly;

- types of physical activity: morning exercises - 45\%; run - 10\%; swimming - 7\%; yoga $10 \%$; fitness - $25 \%$; physiotherapy- $2 \%$.

Analysis of the data allows us to conclude that approximately $55 \%$ of girls and $30 \%$ of boys suffer from physical inactivity; the rest have irregular, low physical activity. There is an obvious need to increase motivation to go in for sports for the prevention of hypodynamia. Consistency, efficiency and an individual approach to each student is possible when communicating with a digital mentor-trainer.

In order to unify the distance training process in the research work, an algorithm was drawn up for increasing physical activity and achieving a certain sports result.

The main blocks are: cardio training, joint gymnastics, stretching, spiritual practices yoga, meditation, relaxation, body architecture - training of muscle groups.

Statistical processing of basic indicators and results achieved in the process of testing the "SportTrainer" system was carried out.

In order to increase physical activity and achieve a certain sports result in the system "SportTrainer " has compiled a calendar plan for testing in control groups, the main stages of which are presented in Table 1.

Table 1. The main stages of development and testing of the "SportTrainer" system

\begin{tabular}{|c|l|}
\hline \multicolumn{2}{|c|}{ ARTIFICIAL INTELLIGENCE SYSTEM"SPORT TRAINER" } \\
\hline \multicolumn{2}{|c|}{ The first stage } \\
\hline $\begin{array}{c}\text { Design of } \\
\text { architecture } \\
\text { information }\end{array}$ & $\begin{array}{l}\text { Design of the structure of storages and databases. } \\
\text { Investigation of video data as a result of the system operation. } \\
\text { Development of algorithms for obtaining images from a video }\end{array}$ \\
\hline
\end{tabular}




\begin{tabular}{|c|l|}
\hline system & $\begin{array}{l}\text { stream. } \\
\text { Development of an image recognition module for building a model } \\
\text { of the human body and its movements in space. } \\
\text { Processing of accumulated data and the formation of models on } \\
\text { their basis for training neural networks. } \\
\text { Development of mathematical models of basic training and } \\
\text { reference movements. } \\
\text { Training and testing of neural networks. }\end{array}$ \\
\hline \multicolumn{1}{|c|}{ The second stage } \\
\hline Design of & $\begin{array}{l}\text { Design of the client side of the information system and interfaces } \\
\text { mobile } \\
\text { for displaying statistics. } \\
\text { Development of the recommendation system module. } \\
\text { Design and development of receiving speech commands from the } \\
\text { user. } \\
\text { Development of dialog control algorithms. } \\
\text { Development of natural language synthesis models. } \\
\text { Development of a feedback generation module. } \\
\text { Testing the operation of the digital system. }\end{array}$ \\
\hline
\end{tabular}

The algorithm for increasing physical activity is shown in the figure 1 .

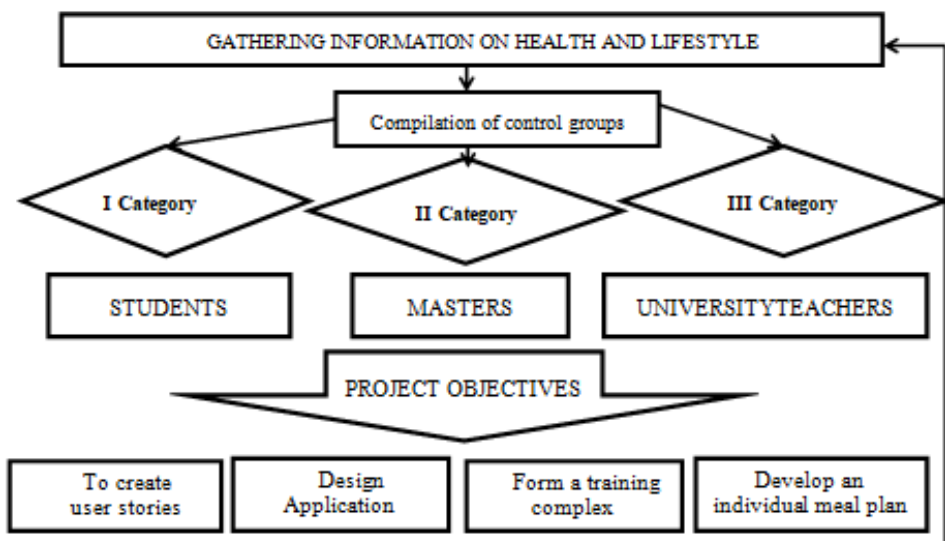

BROADCASTING VIDEO DEMONSTRATORCORRECT EXERCISE TECHNIQUE

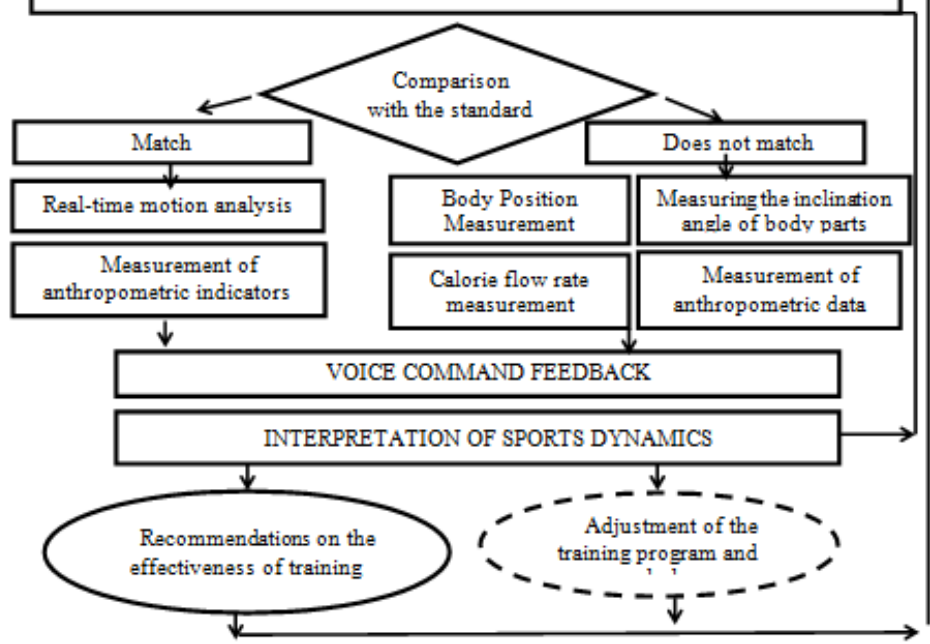

Fig. 1. Algorithm of the curator-trainer in the "SportTrainer" system 
In accordance with the developed algorithm, trainings were conducted in the control groups, the interpretation of the sports dynamics of changes in the intensity of training indicators was carried out, the individual training plan was monitored, and the nutritional balance for the control groups of students was taken into account.

Testing of the "SportTrainer" system in control groups demonstrated the implementation of the following tasks:

- Each participant of the "SportTrainer" system program is provided with full-fledged advice on individual training, proper nutrition, nutritional supplements.

- An intelligent recommendation system automatically selects high-quality nutritional supplements and sports nutrition based on the individual characteristics and goals of the participant at the most favorable prices in Rostov and the region.

- With the help of the "SportTrainer" system, through a webcam, it records the video stream for processing in real time and automatically monitors the correctness of the exercises being performed.

- The "SportTrainer" system demonstrates reference movements to the learner in real time and provides an assessment of the current movements with the addition of corrections from the reference model.

- The "SportTrainer" system provides feedback during training through an audio channel and headphones, supports the synthesis of recommendations in various speech styles.

The "SportTrainer" system provides continuous video processing and keeps track of the student's movements, as well as saves a training record for later analysis.

\section{Conclusion}

The result of the implementation of the project of the "SportTrainer" system, in accordance with the blocks of the algorithm, were:

- development of an individual general health plan of physical activity;

- development of special sports programs aimed at achieving specific sports goals and results;

- recommendations for improving the performance of certain elements of the exercise and adjusting the training program;

- presentations and explanations of the influence of bad habits, physical inactivity, unhealthy diet on indicators of physical and mental health;

Obviously, the ways to overcome physical inactivity should be aimed at increasing physical activity in each person.

It is assumed that the "SportTrainer" system in the mobile application of students and other users will make the process of playing sports more convenient, efficient and cheaper.

\section{References}

1. I.A. Koryagina, G.V. Korolev, Humanities 3 (47), 155-158 (2019)

2. Wikipedia. Available at: https://ru.wikipedia.org

3. A.A. Evdokimova, St. Petersburg Educational Bulletin 3 (3) (2016). Available at: hftps://cyberieninka.ru/articie/n/zdorovyy-obraz-zhizni-studentov-gipodinamiya-i-putieyo-preodoieniya (accessed: 12.03.2021).

4. On the national development goals of the Russian Federation for the period until 2030, Decree of the President of the Russian Federation of July 21, 474 (2020). Available at: https://www.garant.ru/products/ipo/prime/doc/74304210/ 
5. P.Y. Dugnist, V.A. Mil'khin, S.M. Golovin, E.V. Romanova, Human Health, theory and methodology of physical culture and sports 4 (7), 3-25 (2017). URL: http:// journal.asu.ru/zosh/article/view/3463 (In Russ.).

6. E.I. Savko, S.V. Hozhempo, Human Health, theory and methods of physical culture and sports 4 (11), 62-76 (2018). Available at: http://journal.asu.ru/zosh/article/view/4749 (In Russ.).

7. M.R. Arpent'yeva, Human Health, the theory and methodology of physical culture and sports 4 (11), 14-36 (2018). Available at: http:// journal.asu.ru/zosh/article/view/4689 (In Russ.).

8. S.V. Oganesyan, A.L. Grigoryan, Human Health, theory and methods of physical culture and sports 4 (11), 3-13 (2018). Available at: http:// journal.asu.ru/zosh/article/view/4690 (In Russ.).

9. Available at: https://www.coachseye.com

10. Available at: https://www.voltathletics.com)

11. Available at: (https://www.fitnessai.com)

12. Available at: (https://virtuagym.com)

13. S. Beyel, J. Wilhelm, Ch. Mueller, P. Zeile, U. Klein, Der Jahrbuch der Hochschule Bochum 6, 689-699 (2016). DOI: 10.13140/RG.2.1.1451.6721

14. I.Yu. Golovinova, A.A. Merkulova, Physical culture, sports, tourism: problems and prospects 5 (30), (2019).

15. S.A. Litvinov, Theory and practice of social development 8, (2014) Available at: https://cyberieninka.ru/ articie/n/innovatsionnye-zdoroviesberegayuschie-tehnoiogii-vsisteme-fizicheskogo-vospitaniya-vuza.

16. O.P. Piyanzina, T.B. Semenova, Science and Prospects 1, (2017). Available at: https://cyberleninka.ru/article/n7ispolzovanie-informatsionno-kommunikatsionnyhtehnologiy-v-formirovanii-fizicheskogo-razvitiya-v-pedagogicheskoy-deyatelnosti-doo (accessed: 14.03.2021).

17. I.O. Egorochkina, V.O. Ekizyan, Universal product In the collection: Construction. Architecture. Economy: Materials of the International Forum "Victory May 1945": a collection of articles, Ministry of Education and Science of the Russian Federation, Don State Technical University, Trade Union of Public Education and Science Workers of the Russian Federation 28-30. (2018). 